\title{
Téoros
}

Revue de recherche en tourisme

\section{Montréal tu m’habites!}

\section{André Hut}

Volume 10, numéro 3, novembre 1991

Destination Montréal : d'hier à demain

URI : https://id.erudit.org/iderudit/1079206ar

DOI : https://doi.org/10.7202/1079206ar

Aller au sommaire du numéro

Éditeur(s)

Université du Québec à Montréal

ISSN

0712-8657 (imprimé)

1923-2705 (numérique)

Découvrir la revue

Citer ce document

Hut, A. (1991). Montréal tu m’habites! Téoros, 10(3), 58-60.

https://doi.org/10.7202/1079206ar

Ce document est protégé par la loi sur le droit d'auteur. L'utilisation des services d'Érudit (y compris la reproduction) est assujettie à sa politique d'utilisation que vous pouvez consulter en ligne.

https://apropos.erudit.org/fr/usagers/politique-dutilisation/
Cet article est diffusé et préservé par Érudit.

Érudit est un consortium interuniversitaire sans but lucratif composé de l'Université de Montréal, l'Université Laval et l'Université du Québec à Montréal. Il a pour mission la promotion et la valorisation de la recherche. https://www.erudit.org/fr/ 


\section{Euro-Info}

Des amis du Quebecer de Monmeal, le belge Andre Hur, onimateur, joumaliste ef correspondon régulier de Téoros en Europe el le fronçals Bernard Noulin, directeur general de Promrour et delégué general adjoint de Cecorel ont visite Montréal de rnulriples fols ces 20 demieresonnees. Nousleur avons demonde de témoigner de leur artodhementd Montreal, ce qu'ilsont fair aveccholeur, possieer gronde sincerrivé.

\section{Montréal fu m'habites!}

\section{Por André Hut}

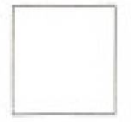

En foir, je ne suis jamals venu d Montreol comme touriste, pour la visiter. Aucontroire, certevilleríérail pour moi que le lieu d'arive ou Quebec er de rerour vers Druxelles a couse de son aeroport a Mirobel. D'ailleurs, les premiers Quebécoisque j"oiconnusmiavaient dir: all ne four pas juger le Québec a partir de Morreal. Levai, le Quebecprotond, Cesren dehors: les Laurentides er le lacsi-Jean, Quebec la CoreNord ou la Gospesie... les Contons de I'Est! c'est pourquoi, opres avoir revu les amis o Monired el regle nos affaires... je lo quirtais choque fois ou plus vire

Er pourtant, depuis press de 14 ans er 18 venues dans la province, Monrélel mía progressivemenr ocatoche molgre mol er a mon insu.., ou point que je souhaite d present y revenir, une fois, pour ellememe ofin de la mieux décou. vir et lo connoirre dons roures ses focertes

\section{Echanges ef stages}

J'a fait portie de l'un des trois premiers stoges gouvernementoux qui inouguraient des ecthanges culnurels priviegies enire la commu. noure francophone de Delgique er le Quebec, en 1978. Le theme: Le rourisme social exerce dons la province. Premiere erope, Montréal, évidemment, avant de partir pour Gronby, Quebec ef RiviereduLoup. Nous logions a l'Auberge de jeunesse, rue Aylmer. afin de prendre noromment contod ovec A. gricolour, sirué d l'epoque boulevard Viger.

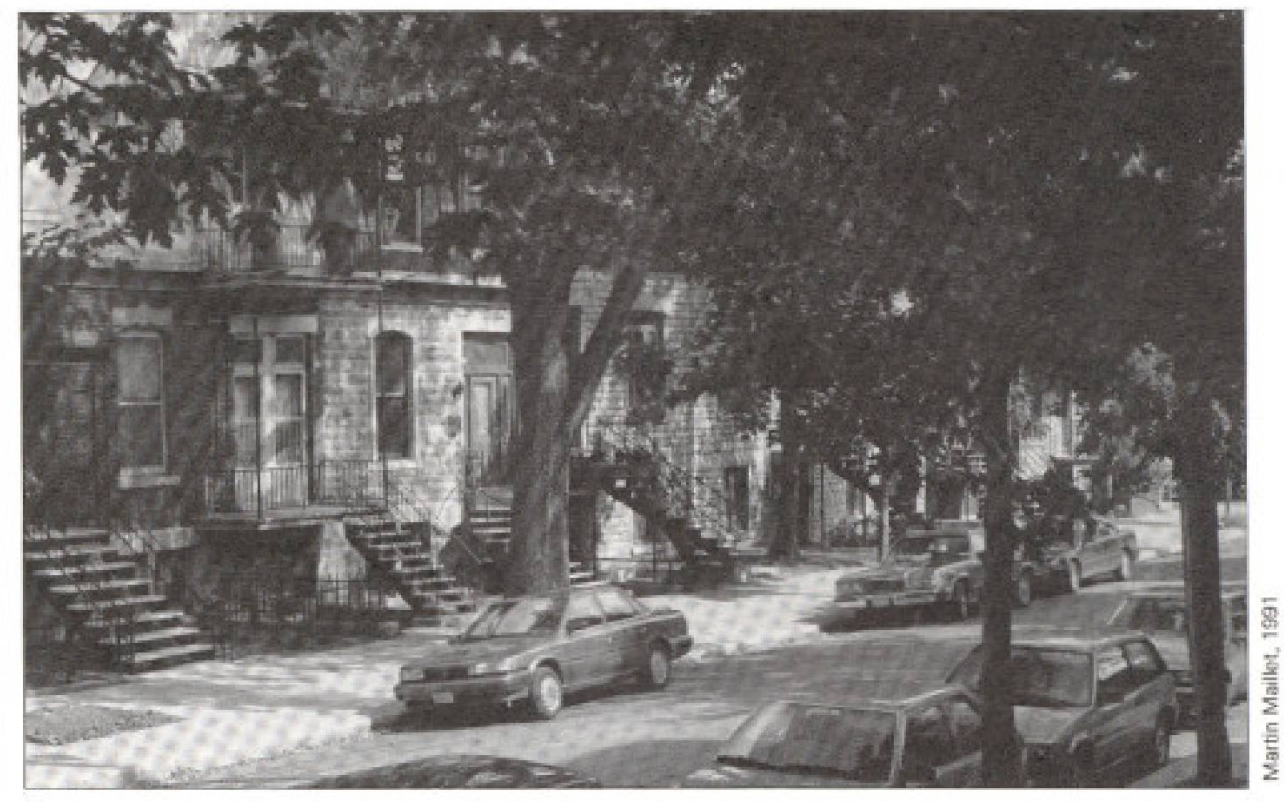

I'Insiitur du tourisme er de l'hotellerie cinsi que le secretarior provincial de liorgonisme qui nous hebergeair. Sur Montréal, les deuxartrocrions rouristiques intemarionales, le Parc olympique er les restes de l'Exposition interno tionale d'lle SreHelene, ne nous apprirent pas grand chose... vu qu'elles ne furent pas resituees dons leur contexte économique, socid, oulrurel er politique, y compris les retombees positives er negarives pour la ville. Nous ap primes quond méme que le mö́r du stade olymplque riovir pos encore pu êrre consuit par manque de fonds, les couts reels des installations olympiques ayont depossé les previsions, ceque l'on reprochair ouverrement ou moire de la ville!

Heureusement que deux ans plus tard, en 1980, l'assemblee generale er le colloque du Dureou internotionol du rourisme social (BITS), eurent lieu a Montreal er a Quebec. J'eus l'occasion de faire connaissonce avec l'un des participants de la wable sectorielle du tou risme: ainsi que du Regroupemen Losir Que bec don elle esr liune des composonres. C'est le debur diune longue, fructueuse er anicole collaborationqui perdure roujoursoujourd hui.

Pour en sovoir plus sur la siruarion sociole er les rélires quoridiennes de la popularion quebe coise - qui expliquent notamment le nondeport en vocances er les obstodes á celuiti. j'obrins : en 1982, un stoged ce propos dans lecodredesaccordsculurels, comme delegué de l'organisorion que jowais fondee ef animais, le CTL (culnure - rourisme - loisirs). Cela me permir de rencontrer. a Montreol, les responsables des arganisations et des comres d'action agissont dons et avec le milieu popu laire: locotoires, thomeurs, consommoteurs, fernmes, crecthes, ploines de jeux, troisieme oge, odolescenrs, groupes erhniques, comps forniliaux Centraide... Montréal a l'ovantoge de regrouper sur son temirire pas mal de secretrorials provincioux de ces organisotions sociooulurelles, de plus, dańs les quartiers populaires, les actions er les lutres socioles éraient, al'epoque, particulierement developpees el vivantes, notomment au Centre-Sud. Celuigi efait d'ailleurs encore troumatise par les consequences sodoles des demolitions de logements anciens, do loyers moderes, dons le bos de la ville... afin d'y consmire la Tour de Rodio-TV Conodo er de gronds boulevards a circulation rapide.

Jy rerrouvais les memes couses er les memes effers qu'a Druxelles fant dans les sinations sociales des hobifonts ogresses de rous cotes que sur le plan d'un urbanisme megalomane souvogeer destrugeur, ateont de vosres deserts urbains, de friches inhobiteses et inhospitalieres une fois que les employes on quirle ces rours de bureoux anomymes.

\section{Géogrophie socio-culturelle}

Le rouriste ordinaire, americain ou europeen, ne connait de Montrél que des sires er monvments: meme le Vieuxhontréal est plutor folkiorique ef commercial qu'une interpreto rion de thistoire economique, sociole el culrurelle de ces lieux privilegiés du posse er de l'ome de lo vile.

Il ignore celte geogrophie militonte de lo vie assoctative, notamment les localisonons de secrérariars provincioux ou urbains d'orgonisotions socio-uliurelles, mentionnees plus hout, occompognees souvent de centres d'information el de documentation: ces der. niers se sinuent un peu portour mois sont sou vent regroupes dons certaines rues el quartiers bien precis. Ils representeroient lo bose 
ditineraires devisites quidees surcetheme. Le Regroupement Loisir Québec, por exemple, fut dobord sirue sur JABRY puis ou Porc oymplque. Lescomires locouxd'action populoire, quont of eux, quodrillent leur quartier respectif comme autant de moillons diune traine de solidorite. J'ai arcule ainsi, à trovers Montréal, pour me rendre dons ces divers lieux significarifs de lo virolite d'une popularion qui se prend en tharge er le destin de so ville, en empruntont d'alleurs les ronsports en comt mun, metro er outobus, mais oussi beoucoup apied, lenezer les yeux ourieuxdes poysoges er des odeurs qui se diwersifient selon les quarriers du bas, du milieu ou du haut de la ville! L'oir respirable er laverdure suivent l'oscersion des dosses sociales du bos vers le haut.

Comme pour Panis, Londres ou Druxelles, les noms des srorions de metro contivent une litonie qui chante dons ma féte el miémeuf qux lomes quand je les rerrove, por hasard. dons un inteview ou une chonson de Quebe cos, a la television, en Delgique, sur la thaine TV5!

Loure géogrophie, celleld, est convivile L'ovantoge des sqges, colloqueser echonges, Cest derencontrer des peronnesqui, exerçont le meme domaine d'ocivires, on ploisir ó portager ovec une certaine complidié cor I| y a une connivence qui oume les boudhes, les cours er les portes. Les $x 000$ porticipants oux echanges ovec le Quebec depuis 10 ans sont des amoureux fous de la province et des Quebecols er representenr, en communouré francaise de Belgique, des ogents publicitaires enthousiostes

En effer, les Québecols sont choleureux er imirent focilement leurs hotes a prendre un por, ovenir souper thez eux a pariciper dune ferte d'onniversoire ovec leurs omises. Du coup je decouve un ourre Montred encore. Non plus seulement des lieux fonctionnels des crgonisations mais les ethanges interperson. nels se deroulant ou domiole, thez Louis ou Nomand L Longueuil ou rue de lo Roche dons le quartier Ahunisic, thez Dione ou Marjolaine rue Fontaine ou Dordeoux dons le quartier Rosemonit thez Richarder Guylaine, rue Notre. Dome oues ou rue Decelles thez Pierre ou Guy, rue Sr-Andre ou Chombord thez Liselene ou Celine! Le merroet lebus miamenentaces divers coins de Montreal mais les arrets ont certe fois une soveur differente, plus chaleureuse encore, dodeurs el de plors miores. homards ou poello de portogeser déthanges plus intimes, dons le serieux de lo confidence ou les rires des souvenirs communs. J'y re frouve doilleurs oussi ceux que j'a ocoueilli, d mon rour, lorsquils son venus ó Druxelles, en reciprocite ou sens fort du terme.

Entin. une outre geographie encore, holque cetre fois quond ces amises montrealais miemmenerent ovec eux dons leurs loisirs: le
Vieuxhontrol quide el wu dravers les yeux d'Hermyle; le feud artifice international sursteHelene ovec Louis, Morie er Julien, pormi lo foule, sur le grand pont le polnt-devve sur Montreal, diou lion operçoir enfin le fleuve. avecMaude communiant as sonodmiration; le concert de diorales d'enfants avec Liseléne. Françols el Patrick o POratoire Saint-Joseph, en pelerinoge alnurel, ou le pique nique au Parc regional du Cop S-Jocques, au bord du SainLourent entin embrosse... II orrive oussi de quilter Montreol pour accompagner o leur villegiarure, sur le bord du loc ensoleille ou la compogne ennelgee, Louis ou Guy er Jocelyne.

\section{Du milieu associatif à l'Université}

J'owais eu evdemment l'occosion, plusieurs fois, de foire poncuellement un expose sur l'onimation touristique dons l'un ou l'outre groupe derudionrs groce do Lous ou Pierre. professeurs o IIUQAM en rourisme ou en parrimaine. Mais le fait de remplocer Louis, en onnee sabbarique, pour domer son cours dinifiation ou fourisme sood er oulfurel, cons. ritue un sesame oume roi, pourentrer de pleinpied dors le milieu universitoire er non plus comme, de l'extérieur. en imite de possoge. Du secterorior du module de gestion er intervention rouristiques (GIT), ocoueilli par des secretures ousi serviobles que sourionres, au locol des charges de cours d la rencontre de nouveouxcollegues de labibliotheque habiree por son responsoble attentionne au service de prér dumaterlel oudiovisuel, en posson par lo cooperative d' othat, le restaurant ou -dine pas manquer pour un belge - les biéres du bor. jusqu'o la dosse ou alrendent 37 erudionts: quel dédale de couloirs el de possoges, d'escaliers ef doscenseurs goce da ce loby rinthe souremoin wante por la publicte touris. rique. En hiver, a pied sec, cest voiment pronique!

Une etapesupplementaire dons la decouverte d'un des milieuk universitaires qui morquent lo veerlideniremontreoloise comme de meime le GI, de son core, s'imbrique dans les realites er les enrreorises rourisiques de la communouté ubaine. Mais qusi un pos de plus dons la connaissance des collegues Morcel, Jean, Marc ou Louise... al'occasion de conversations informelles, de fins de semaine vecuesentemble ou de repas portoges dans les resrourans qui reflerent la presence la gomonomie des. divers groupes ethniques immigres a Monmeol commed Druxelles. Lesorigines geogrophiques er socioles de ces professeurs de meme que celles des erudionrs - revelees por mon enquerte qupres de ces demiers - confirmenr les foçons resdiverses dese situervis-vis de cere metropole dons loquelle ils exercent leur profession ou leurs erudes: pour centoins, por necessire el sans altodie affective a la ville. comme des immigrants de l'interieur; pour d'outres, un hour degre dintensite d'opportenonce er d'engogement, progressif ou tres protond, de lo port de Montréalais d'adoprion ou de naissance!

\section{Une planification de Travenir}

Groce a un periodique - $a$ present disparu regroupont des informations sur les octivites des groupes populaires et progressistes, J'ol eu l'occosion, il y a 10 ans, de participer $\mathrm{a}$ une visite thematique, annoncee er oroonisee por M. Wourers, dons le quartier CentreSud wles façodes el l'ardnirecture typlque des maisons ouvieres du debur du siede: acproche possionnante w la presence dons le groupe de personnes oyonr thobire ces rues jodis ou ayant travallé dons ces entreprises presentement fermées ou réaffectées

Le posse industriel, qui a protondement marqué tant le poysoge que lo vie ou la mémoire colledive des trovailleurs er trowailleuses de l'epoque, redevient percepible oux Montrélois er $d$ ses visireurs groce d des initiatives commel'Ecomuse duFier Monde ouleCentre d'interpretation de I'histoire urbaine d'Hoctielogo Maisonneume. J'ai eu la dhance de renconirer ces personnes er de les ecouter ovec emorion cor je refrouvais, comme un ecto, l'histoire de mes grands parents er des régions ouvieres de Charlerci ou de Liege ou j'ai vecu er travoille notamment poml la jeynesse oumiere: exploitation, thomage, fermerures, lurres syndicoles..!

J'owais tout celo, en tête, quond l'opportunité se presenta d'être present, comme joumaliste a laconference de presseoule nouveou maire er ses colloborateurs presentolent a l'opinion publique un projer de plan direcreur d'urbonisme pour lo vile. J'y reconnoissais certaines oplions qui ovient ere exprimbes dejo lors de reunions preporatoires oux elections-oúl'on mioval entroine-arganisees por les opposonits au maire Dropequ el qui sont ocruellement ou pouvoir.

Volla diverses iniriarives quin tourisme urbain devaitoppyerer developper: rontl'ewocotion du passe qui donne les clefs dinterpretotioner de comprehension sur ce que les visifeurs aperçovent de la ville d'oujourdhui... que la possibiline permonente d'sire informe sur les projets de son developpement futur avec les options qui les soustendent

A ce propos, exprimant dans mon cours, mes regrers que les rouristes nioient pos hobiuellement de relles propositions, que leurs hores ne leur fosent pos decouvir leur quartier de résidence, de fondtions ou mème certaines parties de la vile qu'lls offectionnent portic. lierement, que le Vieux-Port er le fleuve SointLourent soient si peu occessibles, frequenteset opprecies por les Montreoloisque jerencontre. deux erudiants on releve le defil Benoî mia foir visirer le quartier où il est venu resider de 
l'exterieur; la ne ou il hobite, l'ecole, l'arena. le cafe quill frequentait de mentre que les prindpolesentreprisesquiconaderisent l'activite economique decenteportie de laville Liseléne, de son cole, fille dimmigres suisses, ayon realise une efude sur le rourisme d Montreal, miaemmené le long du Sanr-Lourenr d'Est en Quest jusqu'ou Cop 5i-Jocques. Cerait la premiere fois, depuis 14 ans! Cela mia fortement impressionne de decouvir ce liftoral que j'ignorais parce qu'd Bruxelles, nous sommes frumbs de la disporition de notre riviere - la Senne - dons une canalisarion sourerraine de meme que les divers bros de conouxpenerront ou centre de la ville, en patte d'oie, comme d Amsterdom, er rembloye depuis 1905 !! Pourquol les Montrélais que je connais ne miont jomais fait porcourir avec le même enthousiosmedpied, avelo ouenvoifure les berges du fleuve er reveler la beoure er la diversire des maisons er propriétes résidentielles? Les en jeux soooux er economiques? Les menoces ecologiques?

\section{Conclusion}

En definirive, je me rends compte à quel poinjiol ete un privilégie dons certe decou verte de Montreal er de ses hobitonts. J'al profire des reseoux des relations de Rithard er Louis dons lesquels ils miont iniroduir. Com ment les autres tourisres dogrement, de conferences ou d'affaires, selon leurs centres d'inverers, pourcient-ils beneficier des mentres dhonces de renconires er de decouvertes?

Seul un lieu, reperable er diffuse, anime par des personnes ressources du milieu ossociatif, pourrait leur liver les listes d'adresses, les cartes de leurs localisations, les calendriers de leurs octivités ouvertes oux visileurs - fêres, exposirions, visires thematiques guidees-concernont les organisations socioculfurelles provinciales, erthniques er locales, siruees a Montrél? Les odresses er horcires des videorheques, ou lion peur revoir les rémolgnages des evenements qui ont marque son hisioire ou les documenraires qui livent des clefs dinterpretarion des diverses realites el fonctions? Pourquoi les services de presses el de relations publiques de l'Universire, des ministeres provinoloux de Rodio Conoda, de la Ville de Monmeal... riont-ils pos des expositions permonentes presentant leur histoire er leurs projets occompognees de vidéos ou d'exposilions remporaires er themariques, sur des aspects particuliers propres d Montreal? Enfin, ne foudrair-il pas un Cenire dinterpretationsurles relations systemiques du fleuve Saint Lourent er de la Ville de Montreol comme il śen est ouvert un sur lo Loire ou le Cenrre de la Mer a Doulogne, en France? J'oppelle de rous mes voux lo cearion de ces initiatives goges dun tourisme culturel de rencontres er de patages entre les Montrécalais euxmemes, les Quebecois er Conodiens ainsi que les visiteurs etrangers!

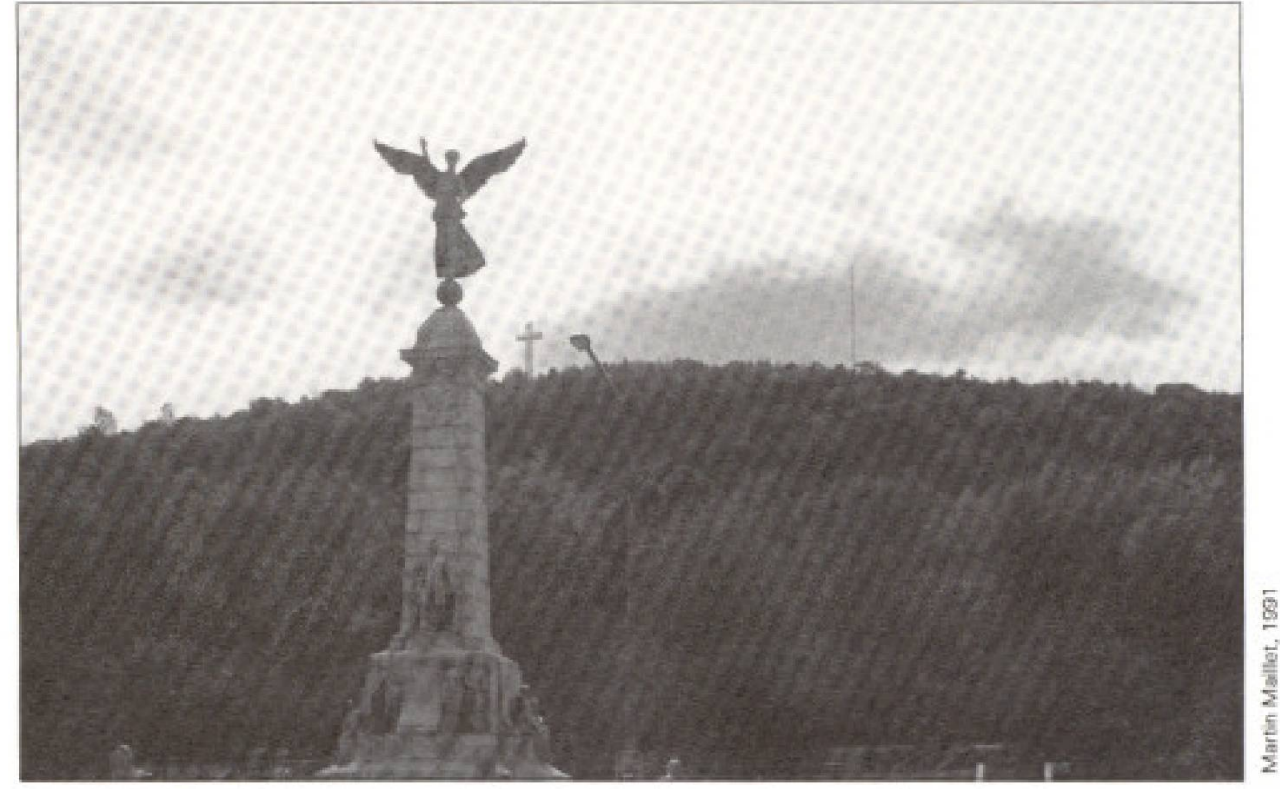

Bonjour ma ville

\section{par Bernard Noulin}

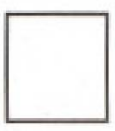

Donjour mo ville - re voild d nouve au plus belle que jamois sous le soleil de cene fin d'ere. Avec lo chaleur, Montreal porfois l"ere tu deviens africaine er, meme si Cartier nía pos voyoge a l'envers de l'hiver, la rue Peel, l'ete. Cest un peu de Modrid ou de Rome a certe epoque.

Pour moi depuis 15 ans, tour commence de la même foçon. Apres Terre-Neuve, deja les locs de la belle province me font signe, deja je sois quiou bour, lorsque lo machine survolero l'outoroure des Laurentides, il y aura Mirabel. J'aime ef je déteste Mirabel. J'aime Mirabel (ron ther ellephont blonc) lorque, dons l'ere roride ou dons l'hiver glacial, simmobilise le monstre. Cest roujours un parcours iniriorique, je nesuis chez moi que lorsque sedecoupe, sur le ciel, ton centreville, la montagne ef I'Orotoire. Decoupoge sublime qui foil que. pour quelques instonts, ru deviens theórre d'ombres, Je dereste Mirabel lessoirs de retour. Mais en cer instont, je ne veux pos en porler.

Souverir mes amis dial ou de labbos (mois ou est id ou est lobas?) me demandent pourquo je t'aime. Pourqual, loin des rives de la Seine. je te irouvesuperbe, toi qui ríapos de Tuileries, de Loume ou de Pontheon.

A vai dire, ru as beoucoup plus. Bien sôr que ru n'os pos ce qui fair le charme de ma ville narole (que joime presque ourani) mois ru as pour moi des imoges fugoces er vives qui, depuis 15 ans, ont risse le fil de ma passion.
Comme un marin doutomne, lorque deja le vent descendu du Labrodor cherche o rengourdir. Tu oscilles olors entre le soleil qu Irve son dernier combar er le froid qui wa re soisir juqu'ou printemps. A cette epoque, tes deux sont superbes, souvent rouges, l'air lim. pide. A ces rouges celestes se melent ceux de la montogne, teintes superbes, melonge de feucomrne pourbrulerune derniêre fols l'eclor de ronérequise meure Cemarinta, llyodejo bien des soisons, j'érais dans un hotel du cenrreville donnani sur le fleuve er le pont Jocques Cartier. Cérair 11 y a longremps er pourtant il reste en ma mémoire. Dentelle de fer se dérachant sur ce ciel rouge. Dons le silence de certe chambre, je voyais le rafic posser le fleuve, ron fleuve er auroni de sujets allont dans la dié, allon vers Derri ou Laurier. ville avalont comme un colosse, ville se nou rissant de ceux de Longueuil ou St-Jean.

Cerair, pelle mêle, un qurre jour sur Coredes. Neiges, un jour d'éte el une pluie borrone thoude, omendue depuis lemorin pour souloger lescorps, les ortres des moux queru infliges $p$ ar res choleurs ront souhailees au coeur de jonvier mais rellemenr erouffanres que porfois res hobironis disent que l'hiver a du bon.

Je ne sais pourquoi celte image est en moi. II pleuvair wo boire debout ${ }_{r}$ les rares passonts pressaient le pos, il pleuvait er, jetais la seul regardant la montagne érincellanre sochoni quid trovers le rideou de plute, mes dhers ecureuilsretrouvilent pouruntemps seulement la plenirude des themins, sentiers er bonos. Jerois ló, la montogne érair belle, lustree, brillante, comme une ve imoginaire offerted ceux qui soven quel remain d'oventure elle codne.

Cérair oussi un dimanche soir en novembre Depuis le morin nous le sovions. Ton ciel erair 